\title{
Hamiltonian Constructions of Kähler-Einstein Metrics and Kähler Metrics of Constant Scalar Curvature
}

\author{
Henrik Pedersen ${ }^{1}$ and Y. Sun Poon ${ }^{2 \star}$ \\ Department of Mathematics, Odense University, DK-5230, Odense M, Denmark \\ 2 Department of Mathematics, Rice University, P.O. Box 1892, Houston, TX 77251, USA
}

Received March 12, 1990; in revised form July 3, 1990

\begin{abstract}
Assuming the existence of a real torus acting through holomorphic isometries on a Kähler manifold, we construct an ansatz for Kähler-Einstein metrics and an ansatz for Kähler metrics with constant scalar curvature. Using this Hamiltonian approach we solve the differential equations in special cases and find, in particular, a family of constant scalar curvature Kähler metrics describing a non-linear superposition of the Bergman metric, the Calabi metric and a higher dimensional generalization of the LeBrun Kähler metric. The superposition contains Kähler-Einstein metrics and all the geometries are complete on the open disk bundle of some line bundle over the complex projective space $\mathbf{P}^{n}$. We also build such Kähler geometries on Kähler quotients of higher cohomogeneity.
\end{abstract}

\section{Introduction}

In this paper, we construct ansätze for Kähler geometries with commuting holomorphic isometries. We express the Kähler-Einstein condition and the condition for constant scalar curvature Kähler metrics as a system of differential equations with respect to the Kähler quotient coordinates and the Hamiltonian functions. This symplectic approach to special Kähler geometry with symmetry has recently been exploited most successfully by LeBrun [7-9]. Also, our concern with Kähler metrics of constant scalar curvature was inspired by the work of Calabi $[2,4]$.

In the case of Kähler metrics with circle action, we solve the equations under some reasonable geometrical assumptions and find, in particular, a family of Kähler geometries with constant negative scalar curvature on the open disk bundle of any complex line bundle $\mathcal{O}(-p)$ over the complex projective space $\mathbf{P}^{n}$ when $p$ is sufficiently large. If one considers the open disk bundle to be differentiably 
isomorphic to the underlying differentiable manifold of the total space of $\mathcal{O}(-p)$, one shall see in Sect. 3 that both the complex structures and the Riemannian metrics are varying within this family. Our family of metrics is in fact almost Einstein and in the limit, when one of the parameters vanishes, we obtain Kähler-Einstein metrics.

We extend our result to metrics of higher cohomogeneity in Sect. 4 by allowing the Kähler quotient to be different from $\mathbf{P}^{n}$. Finally, the case of a higher dimensional torus acting through holomorphic isometries is considered. Readers may consider materials in Sect. 2 and 3 as motivation for, as well as concrete examples of, the general theorems stated in this last section.

\section{The Kähler-Einstein Ansatz}

It is well-known that a Kähler manifold with Kähler form $\Omega$ is Einstein with scalar curvature $2 m \Lambda$ if and only if

$$
\rho=\Lambda \Omega,
$$

where $\rho$ is the Ricci form and $m$ is the complex dimension. This is usually expressed as a system of partial differential equations in the coefficients of the metric with respect to complex coordinates. These equations are most often studied in the realm of the complex Monge-Ampère equation. However, when there are holomorphic isometries, we shall rewrite condition (1.1) in terms of Hamiltonian functions and solve the equations explicitly when there are additional geometric assumptions.

If $T^{N}$ is a real torus acting freely through holomorphic isometries on a Kähler manifold $M$ with Kähler form $\Omega$, then $M$ can be considered as a torus bundle over a real manifold of dimension $2 m-N$, where $m$ is the complex dimension of $M$. The Riemannian metric defines a connection 1 -form $\theta=\left(\theta_{1}, \theta_{2}, \ldots, \theta_{N}\right)$. The fibre coordinate is chosen to be $\left(t_{1}, t_{2}, \ldots, t_{N}\right)$. To choose coordinates on the base manifold, let $X_{i}, i=1,2, \ldots, N$, be the Hamiltonian vector fields generated by this torus action, and $z_{i}$ be the Hamiltonian functions, i.e.

$$
d z_{i}=l_{X_{i}} \Omega .
$$

When $\xi_{\mu}$ are the complex coordinates of the manifold obtained by the Kähler quotient by the torus, $\left(\xi_{\mu}, \bar{\xi}_{\mu}, z_{i}\right)$ forms coordinates on the base manifold. Then the metric on $M$ can be expressed as

$$
g=h+w_{i j} d z_{i} d z_{j}+\left(w^{-1}\right)_{i j} \omega_{i} \omega_{j},
$$

where $h$ is a Kähler metric on the quotient space of each level set of the Hamiltonians and $\omega_{i}=d t_{i}+\theta_{i}$. Note that $\left(w^{-1}\right)_{i j}$ is precisely the inner product between the vector fields $X_{i}, X_{j}$.

To find the Kähler form of this metric, one expresses $h$ as

$$
h=h_{\mu v} d \xi_{\mu} d \bar{\xi}_{v} .
$$

When $I$ is the complex structure,

$$
g\left(I X_{i}, X_{j}\right)=\Omega\left(X_{i}, X_{j}\right)=d z_{i}\left(X_{j}\right) .
$$


Therefore, $-I \omega_{i}=w_{i j} d z_{j}$, i.e.

$$
w_{i j} d z_{j}+i \omega_{i}
$$

are type $(1,0)$-forms. Then the metric can be expressed as

$$
g=h_{\mu \nu} d \xi_{\mu} d \bar{\xi}_{v}+\left(w^{-1}\right)_{k l}\left(w_{l j} d z_{j}+i \omega_{l}\right)\left(w_{k i} d z_{i}-i \omega_{k}\right),
$$

and the Kähler form is

$$
\begin{aligned}
\Omega & =\Omega_{h}+d z_{k} \wedge \omega_{k} \\
& :=\frac{i}{2} h_{\mu \nu} d \xi_{\mu} \wedge d \bar{\xi}_{\nu}+d z_{k} \wedge \omega_{k} .
\end{aligned}
$$

The fact that $\Omega$ is a closed form and that the differential ideal generated by type $(1,0)$-forms is closed is equivalent to a system of differential equations describing the relation between partial derivatives of the coefficients of $\theta_{i}$, the $w_{i j}$ and the $h_{\mu v}$. Due to the fact that we have a Kähler quotient and that the vector fields are commuting holomorphic isometries, these equations can be expressed concisely as

$$
d \theta_{k}=\frac{i}{2} \frac{\partial h_{\lambda \mu}}{\partial z_{k}} d \xi_{\lambda} \wedge d \bar{\xi}_{\mu}+i \frac{\partial w_{k l}}{\partial \xi_{\mu}} d z_{l} \wedge d \xi_{\mu}-i \frac{\partial w_{k l}}{\partial \bar{\xi}_{\mu}} d z_{l} \wedge d \bar{\xi}_{\mu}
$$

Moreover, there is an intrinsic condition that $d d \theta_{k}=0$. This condition can be expressed as

$$
\frac{\partial^{2} h_{\lambda \mu}}{\partial z_{l} \partial z_{k}}+4 \frac{\partial^{2} w_{l k}}{\partial \xi_{\lambda} \partial \bar{\xi}_{\mu}}=0
$$

To derive the condition that the metric is Einstein, recall that

$$
\rho=-i \partial \bar{\partial} \log \operatorname{det} g \text {. }
$$

As $\operatorname{det} g=\operatorname{det} h \operatorname{det} w^{-1}$, set

$$
u=\log \operatorname{det} h-\log \operatorname{det} w,
$$

and let $A=-2 \Lambda$, then (1.1) is equivalent to

$$
d I d u=A \Omega .
$$

Comparing with (1.3), we obtain

$$
\begin{gathered}
4 \frac{\partial^{2} u}{\partial \xi_{\lambda} \partial \bar{\xi}_{\mu}}+\frac{\partial u}{\partial z_{k}}\left(w^{-1}\right)_{k l} \frac{\partial h_{\lambda \mu}}{\partial z_{l}}=A h_{\lambda \mu} \\
\frac{\partial\left(\frac{\partial u}{\partial z_{k}}\left(w^{-1}\right)_{k l}\right)}{\partial \xi_{\lambda}}=0 \\
\frac{\partial\left(\frac{\partial u}{\partial z_{k}}\left(w^{-1}\right)_{k l}\right)}{\partial z_{i}}=A \delta_{i l}
\end{gathered}
$$


In particular,

$$
\frac{\partial u}{\partial z_{k}}\left(w^{-1}\right)_{k l}=A z_{l}+B_{l}
$$

where $B_{l}$ are integration constants, and (1.7) can be simplified using the last equation.

The above discussion then provides a proof for the second part of the following proposition:

Proposition 1.10 (cf. [7]). Let $w$ be a positive definite symmetric matrix and $h a$ positive definite hermitian matrix of smooth functions on an open set $U$ in $\mathbf{C}^{m-N} \times \mathbf{R}^{N}$ which satisfy

$$
\begin{gathered}
4 \frac{\partial^{2} u}{\partial \xi_{\lambda} \partial \bar{\xi}_{\mu}}+\left(A z_{l}+B_{l}\right) \frac{\partial h_{\lambda \mu}}{\partial z_{l}}=A h_{\lambda \mu}, \\
\frac{\partial u}{\partial z_{k}}\left(w^{-1}\right)_{k l}=A z_{l}+B_{l}, \\
\frac{\partial^{2} h_{\lambda \mu}}{\partial z_{l} \partial z_{k}}+4 \frac{\partial^{2} w_{l k}}{\partial \xi_{\lambda} \partial \bar{\xi}_{\mu}}=0 .
\end{gathered}
$$

Suppose also that the closed form

$$
-\frac{1}{2 \pi} F_{k}:=\frac{1}{2 \pi}\left(\frac{i}{2} \frac{\partial h_{\lambda \mu}}{\partial z_{k}} d \xi_{\lambda} \wedge d \bar{\xi}_{\mu}+i \frac{\partial w_{k l}}{\partial \xi_{\mu}} d z_{l} \wedge d \xi_{\mu}-i \frac{\partial w_{k l}}{\partial \bar{\xi}_{\mu}} d z_{l} \wedge d \bar{\xi}_{\mu}\right)
$$

is an integral form for each $k$. Let $M$ be a torus bundle over $U$ such that it has a connection 1-form $\omega=\left(\omega_{1}, \ldots \omega_{N}\right)$ whose curvature is $\left(F_{1}, \ldots F_{N}\right)$. Then

$$
g=h+w_{i j} d z_{i} d z_{j}+\left(w^{-1}\right)_{i j} \omega_{i} \omega_{j}
$$

is a Kähler-Einstein metric with scalar curvature $-m A$.

Conversely, any Kähler-Einstein metric with a torus acting freely through holomorphic isometries, can locally be constructed as above.

Remark. Note that generically (1.13) follows from (1.11) and (1.12) by differentiating (1.11) with respect to $z_{k}$ and substituting (1.12) into the resulting equation.

\section{A Superposition of the Calabi Metric and the Bergman Metric}

In this section, we are looking for metrics with $S^{1}$ symmetry. In this case, Eqs. (1.11), (1.12) and (1.13) in Proposition (1.10), can be simplified as follows:

$$
\begin{gathered}
4 \frac{\partial^{2} u}{\partial \xi_{\lambda} \partial \bar{\xi}_{\mu}}+(A z+B) \frac{\partial h_{\lambda \mu}}{\partial z}=A h_{\lambda \mu}, \\
\frac{\partial u}{\partial z} w^{-1}=A z+B, \\
\frac{\partial^{2} h_{\mu v}}{\partial z^{2}}+4 \frac{\partial^{2} w}{\partial \xi_{\lambda} \partial \bar{\xi}_{\mu}}=0 .
\end{gathered}
$$


Before we begin to find new solutions to these equations, let us look at some examples.

(i) The Bergman Metric on the Ball. This is the standard metric defined in the interior of the unit ball in $\mathbf{C}^{m}$. With a complex coordinate system $\zeta_{1}, \ldots \zeta_{m}$, it can be written as

$$
g=4 \frac{\left(1-\sum \zeta_{\alpha} \bar{\zeta}_{\alpha}\right)\left(\sum d \zeta_{\alpha} d \bar{\zeta}_{\alpha}\right)-\left(\sum \bar{\zeta}_{\alpha} d \zeta_{\alpha}\right)\left(\sum \zeta_{\alpha} d \bar{\zeta}_{\alpha}\right)}{\left(1-\sum \zeta_{\alpha} \bar{\zeta}_{\alpha}\right)^{2}}
$$

We take the rotation

to get

$$
Y=\frac{i}{2} \sum\left(\zeta_{\alpha} \frac{\partial}{\partial \zeta_{\alpha}}-\bar{\zeta}_{\alpha} \frac{\partial}{\partial \bar{\zeta}_{\alpha}}\right)
$$

and

$$
z=\frac{\sum\left|\zeta_{\alpha}\right|^{2}}{1-\sum \zeta_{\alpha} \bar{\zeta}_{\alpha}}
$$

$$
w^{-1}=z+z^{2} \text {. }
$$

Obviously, a generic level set is a sphere and the Kähler quotient is the complex projective space $\mathbf{P}^{m-1}$. Note also that although the Hamiltonian function $z$ is approaching infinity as a point is approaching the boundary of this disk, the disk with its center removed is the unit disk bundle of $\mathcal{O}(-1)$ over $\mathbf{P}^{m-1}$ with the zero section removed. We shall see these phenomena in a more general situation.

(ii) The Multi-Eguchi-Hanson Metrics. This is a family of Ricci-flat Kähler metrics. In order to obtain an $S^{1}$ symmetry, one can consider the MEH metric corresponding to multi-monopoles on a line. More precisely, the metric is

$$
g=V d \mathbf{x} \cdot d \mathbf{x}+V^{-1} \omega^{2},
$$

where $\mathbf{x}=(r \cos \theta, r \sin \theta, x), \omega=d t+\gamma$ such that

$$
d \gamma=* d V
$$

with

$$
V=\sum_{\alpha=0}^{N}\left(r^{2}+\left(x-\lambda_{\alpha}\right)^{2}\right)^{-1 / 2} .
$$

In this case, the rotation field $\partial / \partial \theta$ is a holomorphic isometry.

For the case when $N=1$, set $\lambda_{0}=0, \lambda_{1}=\lambda$, that is the Eguchi-Hanson metric, then the Hamiltonian function and the length of the vector field is

$$
z=\left(r^{2}+x^{2}\right)^{1 / 2}+\left(r^{2}+(x-\lambda)^{2}\right)^{1 / 2}, \quad w^{-1}=\frac{z^{2}-\lambda^{2}}{z} .
$$

Note that the Eguchi-Hanson metric is globally defined on the total space of the canonical bundle over $\mathbf{P}^{1}$. This metric is also known as a Calabi metric.

In view of these examples we impose additional geometric conditions as follows:

(i) the length of the.Hamiltonian vector field is constant on each level set:

(ii) the Kähler quotient metrics obtained from each level set are homothetic. 
We shall see that the Kähler quotient metric is necessarily Einstein under these two conditions.

Remark. If $M$ is compact and $g$ is Kähler-Einstein with positive Ricci curvature then (i) is equivalent to the Kähler quotient metric being Einstein. ([5], p. 106).

Let $q_{\mu \nu}$ be the coefficients of the quotient metric with respect to the coordinates $\xi_{\mu}$. Then condition (ii) implies that

$$
h_{\mu v}\left(z, \xi_{\mu}, \bar{\xi}_{\mu}\right)=f(z) q_{\mu v}\left(\xi_{\mu}, \bar{\xi}_{\mu}\right) .
$$

With condition (i), Eq. (2.3) is equivalent to require

$$
f(z)=C z+D,
$$

for some constants $C, D$. Now,

$$
u=\log \operatorname{det} h-\log \operatorname{det} w=\log \operatorname{det} q+n \log (C z+D)-\log w,
$$

where $n$ is the complex dimension of the quotient space. Then (2.1) is equivalent to

$$
4 \frac{\partial^{2} \log \operatorname{det} q}{\partial \xi_{\lambda} \partial \bar{\xi}_{\mu}}+(A z+B) C q_{\lambda \mu}=A(C z+D) q_{\lambda \mu} .
$$

As a consequence of this equation, $q$ satisfies

$$
-\frac{\partial^{2} \log \operatorname{det} q}{\partial \xi_{\lambda} \partial \bar{\xi}_{\mu}}=k q_{\lambda \mu}
$$

with

$$
-4 k+B C=A D .
$$

Thus $q$ is a Kähler-Einstein metric with scalar curvature 4nk. Moreover, (2.1) is equivalent to (2.5).

The remaining equation is (2.2). Substituting (2.4) into (2.2), we have

$$
(A z+B) w=\frac{n C}{C z+D}-\frac{d \log w}{d z} \text {. }
$$

Let $K=w^{-1}$, then this equation is transformed to be a linear equation in $K$ :

$$
\frac{d K}{d z}+\frac{n C}{C z+D} K=A z+B
$$

and hence

$$
K=(C z+D)^{-n}\left\{\int(A z+B)(C z+D)^{n} d z+E\right\} .
$$

Note that as long as $C \neq 0$, one can assume that $C=1, D=0$ because in this case,

$$
K=\left(z+\frac{D}{C}\right)^{-n}\left\{\int(A z+B)\left(z+\frac{D}{C}\right)^{n} d z+\frac{E}{C^{n}}\right\}
$$

Then we can choose $z+\frac{D}{C}$ to be a Hamiltonian function. 
Therefore, generically, one may assume that $C=1, D=0$, and hence

$$
w=\frac{z^{n}}{\frac{A}{n+2} z^{n+2}+\frac{B}{n+1} z^{n+1}+E} .
$$

To obtain a metric on a bundle, one must also verify that

$$
-\frac{1}{2 \pi} F:=\frac{1}{2 \pi}\left(\frac{i}{2} \frac{\partial h_{\lambda \mu}}{\partial z} d \xi_{\lambda} \wedge d \bar{\xi}_{\mu}+i \frac{\partial w}{\partial \xi_{\mu}} d z \wedge d \xi_{\mu}-i \frac{\partial w}{\partial \bar{\xi}_{\mu}} d z \wedge d \bar{\xi}_{\mu}\right)
$$

is integral. Now,

$$
\begin{aligned}
-\frac{1}{2 \pi} F & =\frac{1}{2 \pi} \frac{i}{2} \frac{\partial h_{\lambda \mu}}{\partial z} d \xi_{\lambda} \wedge d \bar{\xi}_{\mu} \\
& =\frac{1}{2 \pi} \frac{i}{2} \frac{\partial z q_{\lambda \mu}}{\partial z} d \xi_{\lambda} \wedge d \bar{\xi}_{\mu} \\
& =\frac{1}{2 \pi} \frac{i}{2} q_{\lambda \mu} d \xi_{\lambda} \wedge d \bar{\xi}_{\mu}
\end{aligned}
$$

Since $q$ is a Kähler-Einstein metric, this is the first Chern form of the quotient metric $q$. In particular, it is integral. The local construction is finished.

Now we shall study the global nature of these metrics. The metric tensor is given by

$$
g=z q+w d z^{2}+w^{-1} \omega^{2},
$$

where $w$ is given in (2.6) and $\omega=d t+\theta$ with $d \theta=\Omega(q)$, the .Kähler form of $q$. Furthermore, we now assume that $q$ is the Fubini-Study metric

$$
q=4 \frac{\left(1+\sum \zeta_{\alpha} \bar{\zeta}_{\alpha}\right)\left(\sum d \zeta_{\alpha} d \overline{\zeta_{\alpha}}\right)-\left(\sum \bar{\zeta}_{\alpha} d \zeta_{\alpha}\right)\left(\sum \zeta_{\alpha} d \overline{\zeta_{\alpha}}\right)}{\left(1+\sum \zeta_{\alpha} \bar{\zeta}_{\alpha}\right)^{2}}
$$

with constant holomorphic sectional curvature 1. Then (2.5) implies that $B=n+1$. Also since the Fubini-Study metric is Kähler-Einstein and the canonical bundle of $\mathbf{P}^{n}$ is $\mathcal{O}(-(n+1))$ we get

$$
\begin{aligned}
d \theta & =\Omega_{q} \\
& =\frac{2}{n+1} \rho_{q} \\
& =\text { the curvature of } \mathcal{O}(-2) .
\end{aligned}
$$

We shall prove that we have a metric satisfying

$$
\rho_{g}=\Lambda \Omega_{g}
$$

with $\Lambda<0$ and complete on a disk bundle on $\mathbf{P}^{n}$. Let us first prove a necessary condition on the degree of such a bundle and then go on to see that this condition is also sufficient: Let $Z$ be the zero section of $\mathcal{O}(-p) \rightarrow \mathbf{P}^{n}$ and let $K$ be the canonical 
bundle of $\mathcal{O}(-p)$. From the adjunction formula we have

$$
\left.K\right|_{z}=\left.\mathcal{O}(p-(n+1))\right|_{z}
$$

Thus,

$$
\begin{aligned}
p-(n+1) & =\operatorname{degree}(K)=\int_{\mathbf{P}^{1}} c_{1}(K) \\
& =-\int_{\mathbf{P}^{1}} \rho_{g}=-\Lambda \int_{\mathbf{P}^{1}} \Omega>0 .
\end{aligned}
$$

Hence, as a necessary condition we must have $p \geqq n+1$ with equality if and only if $\Lambda=0$. To prove that our family of Kähler-Einstein metrics contains a metric on $\mathcal{O}(-p)$ for all $p$ in the range $p \geqq n+1$ we proceed as follows: Set $z=\frac{1}{4} r^{2}$ and $E=-\left(\frac{a}{2}\right)^{2 n+2}$. Then the metric is given by

$$
g=V^{-1} d r^{2}+\frac{r^{2}}{4} q+\frac{r^{2}}{4} V(d t+\theta)^{2}
$$

where $0 \leqq t \leqq 4 \pi$ and

$$
V=1-\left(\frac{a}{r}\right)^{2 n+2}-\frac{\Lambda}{2(n+2)} r^{2} .
$$

We have apparent singularities at the zeroes of $V$. Now set

$$
U=(n+1)\left(\frac{a}{r}\right)^{2 n+2}-\frac{\Lambda}{2(n+2)} r^{2}
$$

and assume that $\alpha>0$ satisfies

$$
\begin{aligned}
& U(\alpha)=p \in \mathbf{Z} \\
& V(\alpha)=0
\end{aligned}
$$

Then

$$
\begin{aligned}
\alpha^{2} & =2 \Lambda^{-1}(n+1-p), \\
a^{2 n+2} & =\left(2 \Lambda^{-1}(n+1-p)\right)^{n+1} \frac{p+1}{n+2} .
\end{aligned}
$$

Then from (2.12) we again see that if $\Lambda<0$ we need $p \geqq n+2$. Restrict attention to fibres of the Hopf map $\mathbf{C}^{n+1}-\{0\} \rightarrow \mathbf{P}^{n}$, then $g$ becomes

$$
\left.g\right|_{\text {fiber }}=V^{-1} d r^{2}+\frac{r^{2}}{4} V d t^{2}
$$

where $a^{2 n+2}$ is given by (2.13). Now dividing out by the action of $\mathbf{Z}_{p}$, introducing a new angular coordinate $\tau=\frac{p t}{2}$, and simultaneously introducing a new radial coordinate $R^{2}=r^{2} V$, the fiber-wise metric becomes 


$$
\begin{aligned}
\left.g\right|_{\text {fiber }} & =U^{-2}\left(1+U^{-1} R^{2} r^{-2}\right)^{-2} d R^{2}+\frac{R^{2}}{p^{2}} d \tau^{2} \\
& \approx U^{-2}\left(1-2 U^{-1} R^{2} r^{-2}\right) d R^{2}+\frac{R^{2}}{p^{2}} d \tau^{2} \\
& \approx \frac{1}{p^{2}}\left(d R^{2}+R^{2} d \tau^{2}\right) .
\end{aligned}
$$

Thus, the metric extends smoothly across the origin $R=0$. Finally, note that

$$
\frac{d V}{d r}=\frac{2 n+2}{r^{2 n+3}} a^{2 n+2}-\frac{\Lambda}{n+2} r>0, \text { for } r>0,
$$

so the metric is non-singular for all $r>\alpha=\left(2 \Lambda^{-1}(n+1-p)\right)^{1 / 2}$.

Again $Z$ denotes the zero section of $\mathcal{O}(-p) \rightarrow \mathbf{P}^{n}$. Let

$$
\begin{aligned}
U_{i} & =\left\{\left(z_{0}, \ldots, z_{n}\right) \in \mathbf{P}^{n} \mid z_{i} \neq 0\right\}, \\
V_{i} & =\left\{\left(z_{0}, \ldots, z_{n}\right) \in\left(\mathbf{C}^{n+1}-\{0\}\right) / \mathbf{Z}_{p} \mid z_{i} \neq 0\right\},
\end{aligned}
$$

$i=0,1, \ldots, n$. Then consider the isomorphisms $h_{i}: V_{i} \rightarrow U_{i} \times(\mathbf{C}-\{0\})$, where

$$
h_{i}\left(z_{0}, \ldots, z_{n}\right)=\left(\frac{z_{0}}{z_{i}}, \ldots, \wedge, \ldots, \frac{z_{n}}{z_{i}}, z_{i}^{p}\right)=\left({ }_{i} \zeta_{k, i} \eta\right),
$$

$k \neq i, 0 \leqq k \leqq n$. Then since

$$
{ }_{j} \eta={ }_{i} \eta\left({ }_{i} \zeta_{j}\right)^{p},
$$

and $U_{i} \times(\mathbf{C}-\{0\})$ covers $\mathcal{O}(-p) \backslash Z$ we have an isomorphism

$$
\left(\mathbf{C}^{n+1}-\{0\}\right) / \mathbf{Z}_{p} \cong \mathcal{O}(-p) \backslash Z,
$$

and by sending $Z$ to $\{0\}$ we get a desingularization $\mathcal{O}(-p) \rightarrow \mathbf{C}^{n+1} / \mathbf{Z}_{p}$.

The two processes above of desingularization are compatible: On the fiber of $\mathbf{C}^{n+1}-\{0\} \rightarrow \mathbf{P}^{n}$ we have

so we get

$$
I d z=w^{-1} d \tau
$$

$$
I d R=(V+U) R \frac{1}{2} d t \approx R d \tau .
$$

Then $\eta \approx R e^{i \tau}$ and we have proved that our metric is defined in a neighbourhood of the zero section of the bundle $\mathcal{O}(-p)$ over $\mathbf{P}^{n}$. As the metric is defined for all $r \geqq \alpha$, the metric is complete on a manifold diffeomorphic to the total space of $\mathcal{O}(-p)$. However, one can see that, when $\Lambda<0$, the Kähler metric is only defined on an open disk bundle of $\mathcal{O}(-p)$ over $\mathbf{P}^{n}$ as follows:

We have seen that the fibre-wise metric is given by

$$
\left.p^{2} g\right|_{\text {fibre }}=p^{2} w d z^{2}+4 w^{-1} d \tau^{2}
$$

where $w$ is given in (2.6), with $B=n+1, A \geqq 0, E \leqq 0$. This is a metric on the complex plane such that $\tau$ is the angle-coordinate. Therefore, by uniformization, there is a function $\rho$ of $z$ and a function $g$ of $\rho$ such that

$$
p^{2} w d z^{2}+4 w^{-1} d \tau^{2}=g^{2}(\rho)\left(d \rho^{2}+\rho^{2} d \tau^{2}\right) .
$$


Then

$$
\frac{d \rho}{d z}=p \frac{w^{1 / 2}}{g}, \quad 4 w^{-1}=\rho^{2} g^{2} .
$$

Differentiating the second equality, one has

$$
4 \frac{d w^{-1}}{d z}=2 g \rho^{2} \frac{d g}{d z}+2 \rho g^{2} \frac{d \rho}{d z}=4\left(w^{-1} \frac{2}{g} \frac{d g}{d z}+p\right) \text {. }
$$

Therefore,

$$
\frac{d \log \left(g^{2} w\right)}{d z}=-p w
$$

so

$$
g^{2} w=C \exp \left\{-p \int_{\alpha}^{z} w d z\right\}
$$

where $C$ is a positive constant. Then

$$
\rho^{2}=4 g^{-2} w^{-1}=4 C^{-1} \exp \left\{p \int_{\alpha}^{z} w d z\right\} .
$$

As

$$
w^{-1}=z+\frac{E}{z^{n}}+\frac{A}{n+2} z^{2}
$$

for any given large positive constant $C_{0}$, there is $z_{0} \geqq \alpha$, such that for all $z \geqq z_{0}$,

$$
w^{-1}=z+\frac{E}{z^{n}}+\frac{A}{n+2} z^{2} \geqq \frac{A}{n+2} z^{2}+C_{0}^{2} .
$$

Then

$$
\begin{aligned}
\int_{\alpha}^{z} w d z & =\int_{\alpha}^{z_{0}} w d z+\int_{z_{0}}^{z} w d z \\
& \leqq \int_{\alpha}^{z_{0}} w d z+\int_{z_{0}}^{z} \frac{d z}{n+2} z^{2}+C_{0}^{2} \\
& =\int_{\alpha}^{z_{0}} w d z+\frac{A_{0}}{C_{0}^{2}}\left(\arctan \frac{z}{A_{0}}-\arctan \frac{z_{0}}{A_{0}}\right)
\end{aligned}
$$

where $A_{0}=C_{0} \sqrt{\frac{(n+2)}{\mathrm{A}}}$. Now, one can see that as $z$ is approaching infinity, $\rho$ is approaching a positive constant, i.e. $\rho$ is a bounded function of $z$ and hence the metric on each fibre is defined only on an open disk. This is precisely a generalization of the phenomena of the Bergman metric. However, our metrics can be extended over the zero section which gives the existence in the following proposition. 
Proposition 2.14. For any integer $p \geqq n+2$, the open disk bundle of $\mathcal{O}(-p) \rightarrow \mathbf{P}^{n}$ admits a complete Kähler-Einstein metric with negative scalar curvature. Up to multiplication by an overall constant, there is exactly one such metric which is $U(1) \times S U(n+1)$ invariant.

Proof. The invariance forces the length $w^{-1}$ of the symmetry only to depend on the Hamiltonian function $z$ and then the uniqueness follows from the unique solution in (2.6) of the differential equation.

Remark. B. Bergery has produced a series of Kähler-Einstein metrics of cohomogeneity one on complex line bundles ([1], p. 276). From the symmetry and uniqueness our metric must correspond to an object in his work. However, B. Bergery claims that his Kähler-Einstein metrics are defined on a line bundle. This can only be true as a Riemannian metric.

Remark. If we set $\Lambda=0$ in the metric, we get a Ricci-flat metric on the canonical bundle of $\mathbf{P}^{n}$. This metric has previously been discovered by Calabi in [3]. Also, if $a=0$ we get the Bergman metric. This we also see by comparing (2.6) with the first example. Thus the family of metrics is a kind of non-linear superposition of the Calabi metric and the Bergman metric. For $n=1$ see also [11].

\section{The Constant Scalar Curvature Kähler Ansatz}

In [6], LeBrun constructed a family of Kähler metrics with vanishing scalar curvature. The metrics in this family are complete on complex line bundles on $\mathbf{P}^{1}$. The metrics are not Einstein but the conformal class contains an Einstein metric on the ball in $\mathbf{R}^{4}$ previously found by Pedersen [10]. In this section we shall construct a family of Kähler metrics with constant scalar curvature and complete on disk bundles over $\mathbf{P}^{n}$. This family of metrics contains a higher dimensional generalization of the LeBrun metric.

To obtain the ansatz for constant scalar curvature Kähler metrics with symmetry, one essentially performs a contraction on Eq. (1.11), (1.12) and get the following:

Proposition 3.1. Let $w$ be a positive definite symmetric matrix and $h$ a positive definite hermitian matrix of smooth functions on an open set $U$ in $\mathbf{C}^{m-N} \times \mathbf{R}^{N}$ which satisfy

$$
\begin{gathered}
\left(4 \frac{\partial^{2} u}{\partial \xi_{\lambda} \partial \bar{\xi}_{\mu}}+\frac{\partial u}{\partial z_{k}}\left(w^{-1}\right)_{k l} \frac{\partial h_{\mu v}}{\partial z_{l}}\right) h^{\lambda \mu}+\frac{\partial \frac{\partial u}{\partial z_{k}}\left(w^{-1}\right)_{k l}}{\partial z_{l}}=m A \\
\frac{\partial^{2} h_{\lambda \mu}}{\partial z_{l} \partial z_{k}}+4 \frac{\partial^{2} w_{l k}}{\partial \xi_{\lambda} \partial \bar{\xi}_{\mu}}=0 .
\end{gathered}
$$

Suppose also that the closed form

$$
-\frac{1}{2 \pi} F_{k}:=\frac{1}{2 \pi}\left(\frac{i}{2} \frac{\partial h_{\lambda \mu}}{\partial z_{k}} d \xi_{\lambda} \wedge d \bar{\xi}_{\mu}+i \frac{\partial w_{k l}}{\partial \xi_{\mu}} d z_{l} \wedge d \xi_{\mu}-i \frac{\partial w_{k l}}{\partial \bar{\xi}_{\mu}} d z_{l} \wedge d \bar{\xi}_{\mu}\right)
$$

is an integral form for each $k$. Let $M$ be a torus bundle over $U$ such that it has a 
connection 1-form $\omega=\left(\omega_{1}, \ldots, \omega_{N}\right)$ whose curvature is $\left(F_{1}, \ldots F_{N}\right)$. Then

$$
g=h+w_{i j} d z_{i} d z_{j}+\left(w^{-1}\right)_{i j} \omega_{i} \omega_{j}
$$

is a Kähler metric with constant scalar curvature $-m A$.

Conversely, any constant scalar curvature Kähler metric with a torus acting freely through holomorphic isometries, can locally be constructed as above.

Now, consider the $S^{1}$ case. We assume we have a Kähler metric with one holomorphic symmetry in complex dimension $n+1$ and we assume the length of the symmetry is constant on each level set and that the quotient metrics obtained from each level set are homothetic. From Sect. 2 we know that such a metric has the local form

$$
g=h+w d z^{2}+w^{-1} \omega^{2}
$$

where

$$
h=z q,
$$

and $q$ is the metric on the Kähler quotient. Furthermore, the quotient metric is assumed to be Einstein with scalar curvature $4 n k$ so that

$$
-\frac{\partial^{2} \log \operatorname{det} q}{\partial \xi_{\lambda} \partial \bar{\xi}_{\mu}}=k q_{\lambda \mu} .
$$

Under these assumptions the equation for constant scalar curvature (3.2) is equivalent to

$$
z \frac{\partial \phi}{\partial z}+n \phi=(n+1) A+4 k n
$$

where

$$
\phi=\frac{\partial u}{\partial z} w^{-1}
$$

Solving this equation, we get

$$
w^{-1}=\frac{\left(\frac{A}{n+2}\right) z^{n+2}+\left(\frac{4 k}{n+1}\right) z^{n+1}+G z+E}{z^{n}},
$$

where $G$ and $E$ are integration constants.

To study the global properties of this Kähler metric we proceed as in Sect. 2. We fix the quotient metric to be the Fubini-Study metric with constant holomorphic sectional curvature 1. Furthermore we set $z=\frac{1}{4} r^{2}$ and

$$
V=1-\frac{E}{r^{2 n+2}}+\frac{H}{r^{2 n}}-\frac{\Lambda r^{2}}{2(n+2)},
$$

where $E, H$ and $\Lambda$ are constants. Then the metric takes the following form:

$$
g=V^{-1} d r^{2}+\frac{r^{2}}{4} q+\frac{r^{2}}{4} V(d t+\theta)^{2},
$$


where $\omega=d t+\theta$ and $d \theta=$ curvature of $\mathcal{O}(-2)$. Now set

$$
\begin{gathered}
U(r)=\frac{(n+1) E}{r^{2 n+2}}-\frac{n H}{r^{2 n}}-\frac{\Lambda r^{2}}{2(n+2)}, \\
E=k_{1} a^{2 n+2} ; \quad H=-k_{2} a^{2 n},
\end{gathered}
$$

divide out by the action of $\mathbf{Z}_{p}$ and introduce $\tau=\frac{p t}{2}$; then we may proceed in much the same way as in Sect. 2, using $V$ and $U$ as in (3.9) and (3.11), to resolve the apparent singularity, and to show that the metric is complete on the open disk bundle of $\mathcal{O}(-p) \rightarrow \mathbf{P}^{n}$. Thus we obtain the following proposition.

Proposition 3.13. Let $p$ be an integer and $k_{1}$ and $k_{2}$ be non-negative numbers such that

$$
\begin{aligned}
(n+1) k_{1}+n k_{2}-p & =\frac{\Lambda a^{2}}{2(n+2)}, \\
1-k_{1}-k_{2} & =\frac{\Lambda a^{2}}{2(n+2)}, \\
k_{1}+k_{2} & >1 .
\end{aligned}
$$

Then (3.9), (3.10) and (3.12) define a complete Kähler metric with constant negative scalar curvature on the open disk bundle of $\mathcal{O}(-p) \rightarrow \mathbf{P}^{n}$. This is the unique family of such metrics which is $U(1) \times S U(n+1)$ invariant.

Remark. When the parameter $G$ in (3.8), or equivariantly the $k_{2}$ in Proposition (3.12), is small, the metric is almost Einstein. In fact, the Ricci form is

$$
\rho=\Lambda \Omega-2 G\left(\frac{1}{z^{n}} \Omega_{q}-\frac{n}{z^{n+1}} d z \wedge \omega\right) \text {. }
$$

Then

$$
\|\rho-\Lambda \Omega\|^{2}=4 G^{2}\left(\frac{1}{z^{2 n}}\left\langle\Omega_{q}, \Omega_{q}\right\rangle-\frac{2 n}{z^{2 n+1}}\left\langle\Omega_{q}, d z \wedge \omega\right\rangle+\frac{n^{2}}{z^{2 n+2}}\langle d z \wedge \omega, d z \wedge \omega\rangle\right) .
$$

As $\left\langle\Omega_{q}, \Omega_{q}\right\rangle$ is constant, $d z \wedge \omega$ has length equal to $1, \Omega_{q}$ and $d z \wedge \omega$ are orthogonal and $z$ is bounded away from zero, there is a constant $C$ such that

$$
\|\rho-\Lambda \Omega\|^{2} \leqq C G^{2} \text {. }
$$

In particular, if $G=0$, the metric is the Einstein metric in Proposition (2.14).

Remark. For $\Lambda=0$, we obtain a scalar flat Kähler metric when

$$
k_{1}=p-n, \quad k_{2}=(n+1)-p,
$$

and this metric is in fact complete on the entire total space of $\mathcal{O}(-p)$ :

Proposition 3.14. For any integer $p \geqq n$, the bundle $\mathcal{O}(-p) \rightarrow \mathbf{P}^{n}$ admits a complete Kähler metric with vanishing scalar curvature. Up to multiplication by an overall constant, there is exactly one such metric which is $U(1) \times S U(n+1)$ invariant.

Remark. For $n=1$, this scalar flat Kähler metric is the LeBrun metric. Thus the 
metric in Proposition (3.13) may be thought of as a non-linear superposition of the Calabi metric, the Bergman metric and a higher dimensional version of the LeBrun metric. Also for $n=1$, the LeBrun metric is locally conformal to an Einstein metric. However, we have the following proposition:

Proposition 3.15. For $n \geqq 2$, the constant scalar curvature Kähler metric in (3.13) is conformally equivalent to an Einstein metric of cohomogeneity 1 only when it degenerates into the Calabi metric. For $n=1$, only the LeBrun metric is locally conformally Einstein with cohomogeneity 1.

Proof. Let $Z$ be the tracefree part of the Ricci tensor of our metric. When $\hat{g}=\varphi^{-2} g$ is an Einstein metric, then

$$
Z \varphi+2 n\left(\nabla d \varphi+\frac{\Delta \varphi}{2(n+1)} g\right)=0
$$

where the covariant derivative and the Laplacian are associated to the metric in (3.13). Now, we have $I d z=w^{-1} \omega$ and $Z(X, Y)=-\rho(I X, Y)$, where $\rho$ is the Ricci form. Then we obtain

$$
Z=\left(\frac{n G}{2 z^{n+1}}-\frac{1}{2} A\right)\left(w d z^{2}+w^{-1} \omega^{2}\right)-\left(\frac{G}{2 z^{n+1}}+\frac{1}{2} A\right) z q,
$$

where $w^{-1}$ is given in (3.8). The property of cohomogeneity one forces $\varphi$ to depend only on $z$ and we get

$$
\begin{aligned}
\Delta \varphi= & -\left(\frac{d^{2} \varphi}{d z^{2}} w^{-1}+\frac{d \varphi}{d z}\left(\frac{d w^{-1}}{d z}+\frac{n}{z} w^{-1}\right)\right), \\
\nabla d \varphi= & \left(\frac{d^{2} \varphi}{d z^{2}} w^{-1}+\frac{1}{2} \frac{d \varphi}{d z} \frac{d w^{-1}}{d z}\right) w d z^{2} \\
& +\frac{1}{2} \frac{d \varphi}{d z} \frac{d w^{-1}}{d z} w^{-1} \omega^{2}+\frac{1}{2} z^{-1} w^{-1} \frac{d \varphi}{d z} z q
\end{aligned}
$$

Then (3.16) is a system of three equations

$$
\begin{gathered}
\left(\frac{n G}{2 z^{n+1}}-\frac{1}{2} A\right) \varphi+2 n\left(\frac{d^{2} \varphi}{d z^{2}} w^{-1}+\frac{1}{2} \frac{d \varphi}{d z} \frac{d w^{-1}}{d z}\right) \\
-\frac{n}{n+1}\left(\frac{d^{2} \varphi}{d z^{2}} w^{-1}+\frac{d \varphi}{d z}\left(\frac{d w^{-1}}{d z}+\frac{n}{z} w^{-1}\right)\right)=0 \\
-\left(\frac{G}{2 z^{n+1}}+\frac{1}{2} A\right) \varphi+n \frac{d \varphi}{d z} z^{-1} w^{-1}-\frac{n}{n+1}\left(\frac{d^{2} \varphi}{d z^{2}} w^{-1}+\frac{d \varphi}{d z}\left(\frac{d w^{-1}}{d z}+\frac{n}{z} w^{-1}\right)\right)=0 \\
\left(\frac{n G}{2 z^{n+1}}-\frac{1}{2} A\right) \varphi+n \frac{d \varphi}{d z} \frac{d w^{-1}}{d z}-\frac{n}{n+1}\left(\frac{d^{2} \varphi}{d z^{2}} w^{-1}+\frac{d \varphi}{d z}\left(\frac{d w^{-1}}{d z}+\frac{n}{2} w^{-1}\right)\right)=0 .
\end{gathered}
$$

Now, subtracting (3.22) from (3.20) we get $\frac{d^{2} \varphi}{d z^{2}}=0$, so $\varphi(z)=a z+b$. But if we sub- 
stitute this into (3.21), we see that $A=0$ and that for $n \neq 1$ this equation will only be satisfied if $G=0$, i.e. if the metric is the Calabi metric.

\section{Further Applications of the Ansätze}

(i) Higher Cohomogeneity. In all the constructions above, we fixed the Kähler quotient to be $\mathbf{P}^{n}$ with the Fubini-Study metric. This was done to make the examples more explicit and transparent. However, we can also build metrics on non-homogenous Kähler quotients to obtain metrics of higher cohomogeneity. Thus, let $X$ be Kähler-Einstein of complex dimension $n$ with metric $q$. We assume that

$$
\rho_{q}=\Lambda_{q} \Omega_{q}
$$

with $\Lambda_{q}=\frac{1}{2} l \varepsilon, \varepsilon=-1,0$, or 1 . Here $l$ is the index of $X$ in case $\Lambda_{q} \neq 0$, i.e. the biggest positive integer such that there exist a line bundle $\mathbf{L}$ on $X$ with $\mathbf{L}^{l}$ isomorphic to the canonical bundle of $X$. In the Ricci-flat case, we shall assume that $q$ is a Hodge metric on $X$ and then $\mathbf{L}$ is the line bundle represented by the Hodge form. Again, let $g$ be the metric

$$
g=V^{-1} d r^{2}+\frac{r^{2}}{4} q+\frac{r^{2}}{4} V(d t+\theta)^{2}
$$

where

$$
V=\frac{l \varepsilon}{n+1}-\frac{k_{1} a^{2 n+2}}{r^{2 n+2}}-\frac{k_{2} a^{2 n}}{r^{2 n}}-\frac{\Lambda r^{2}}{2(n+2)} .
$$

Furthermore, let $p$ be an integer and $k_{1}, k_{2}$ be non-negative numbers such that

$$
\begin{aligned}
(n+1) k_{1}+n k_{2}-p & =\frac{\Lambda a^{2}}{2(n+2)}, \\
\frac{l \varepsilon}{n+1}-k_{1}-k_{2} & =\frac{\Lambda a^{2}}{2(n+2)}, \\
k_{1}+k_{2} & >\frac{l \varepsilon}{n+1} .
\end{aligned}
$$

Then we get

Proposition 4.3. Let the parameters satisfy (4.2). Suppose that $\Lambda<0, k_{1}>0$ and $k_{2}>0$, then $g$ is a Kähler metric with constant negative scalar curvature and complete on the open disk bundle of $\mathbf{L}^{l \varepsilon} \rightarrow X$.

We also obtain the special cases of Einstein metrics and scalar flat metrics:

Proposition 4.4. Let the parameters satisfy (4.2). Suppose that $\Lambda<0, k_{1}>0$ and $k_{2}=0$, then $g$ is a Kähler-Einstein metric with negative scalar curvature and complete on the open disk bundle of $\mathbf{L}^{l \varepsilon} \rightarrow X$.

Proposition 4.5. Let $\Lambda=0, \varepsilon=1 ; k_{1}=p-n, k_{2}=n+1-p$ and $p$ be an integer such 
that $p \geqq n$, then $g$ is a scalar flat Kähler metric complete on $\mathbf{L}^{p} \rightarrow X$. For $p=n+1$, the metric is Ricci-flat.

(ii) Torus Symmetry. A trivial way to obtain Kähler-Einstein metrics with $S^{1} \times S^{1}$ symmetry is to take the product of the metrics which were constructed in Sect. 2. In this case, the Kähler quotient is the product of Kähler-Einstein metrics. Similarly, we choose an $S^{1} \times S^{1}$ action on the complex disk with dimension $n_{1}+n_{2}+2$ so that one $S^{1}$ is rotation in the first $n_{1}+1$ coordinates and acts trivially on the rest, another $S^{1}$ acts in the complementary way, then the Kähler quotient is a product of Fubini-Study metrics on $\mathbf{P}^{n_{1}} \times \mathbf{P}^{n_{2}}$. Motivated by these two examples, we shall find solutions to the Einstein ansatz with the following assumptions:

(a) the matrix $w$ is a function of $z_{1}, z_{2}$ only; (b) the Kähler quotient metric obtained from any level set has the form

$$
h=f_{1}\left(z_{1}, z_{2}\right) q_{\mu \nu}^{1} d \xi^{\mu} d \xi^{v}+f_{2}\left(z_{1}, z_{2}\right) q_{\alpha \beta}^{2} d \zeta^{\alpha} d \zeta^{\beta},
$$

where $q^{1}, q^{2}$ are Kähler-Einstein metrics on manifolds of dimension $n_{i}$ and scalar curvature $4 n_{i} k_{i}$.

As a consequence of (1.13), condition (a) and (b) are compatible only if $f_{1}, f_{2}$ are affine functions of $z_{1}$ and $z_{2}$. We set $f_{i}=z_{i}$. i.e.,

$$
h=z_{1} q^{1}+z_{2} q^{2} \text {. }
$$

As

$$
\begin{aligned}
u & =\log \operatorname{det}\left(h w^{-1}\right) \\
& =\log \operatorname{det} q^{1}+\log \operatorname{det} q^{2}+\log \left(z_{1}^{n_{1}} z_{2}^{n_{2}} \operatorname{det} K\right),
\end{aligned}
$$

where $K=w^{-1},(1.11)$ is equivalent to require $n_{i} B_{i}$ to be the scalar curvature of the Kähler-Einstein metric $q^{i}$. Finally, (1.12) is a system of two equations in the entries of the matrix $K$. Assuming that $K_{11}$ depends on $z_{1}$ and $K_{22}$ depends on $z_{2}$ only, this system can be simplified as follows:

$$
\begin{aligned}
& \left(\frac{\partial K_{11}}{\partial z_{1}}+\frac{n_{1}}{z_{1}} K_{11}-\left(A z_{1}+B_{1}\right)\right) K_{22}=\frac{\partial K_{12}^{2}}{\partial z_{1}}+\frac{n_{1}}{z_{1}} K_{12}^{2}-K_{12}\left(A z_{2}+B_{2}\right), \\
& \left(\frac{\partial K_{22}}{\partial z_{2}}+\frac{n_{2}}{z_{2}} K_{22}-\left(A z_{2}+B_{2}\right)\right) K_{11}=\frac{\partial K_{12}^{2}}{\partial z_{2}}+\frac{n_{2}}{z_{2}} K_{12}^{2}-K_{12}\left(A z_{1}+B_{1}\right) .
\end{aligned}
$$

When $K_{12}=0$, one obtains the product of metrics constructed in Sect. 2 .

The matrix

$$
K=\left(\begin{array}{cc}
z_{1}+z_{1}^{2} & z_{1} z_{2} \\
z_{1} z_{2} & z_{2}+z_{2}^{2}
\end{array}\right)
$$

is another solution. This solution corresponds to the Bergman metric.

However, there is a family of non-trival solution obtained by solving the following systems:

$$
\begin{aligned}
& \frac{\partial K_{11}}{\partial z_{1}}+\frac{n_{1}}{z_{1}} K_{11}=A z_{1}+B_{1} ; \\
& \frac{\partial K_{22}}{\partial z_{2}}+\frac{n_{2}}{z_{2}} K_{22}=A z_{2}+B_{2} ;
\end{aligned}
$$




$$
\begin{aligned}
& 2 \frac{\partial K_{12}}{\partial z_{1}}+\frac{n_{1}}{z_{1}} K_{12}=A z_{2}+B_{2} ; \\
& 2 \frac{\partial K_{12}}{\partial z_{2}}+\frac{n_{2}}{z_{2}} K_{12}=A z_{1}+B_{1} .
\end{aligned}
$$

This system has solutions only if $n_{1}=n_{2}$, say it is $n$. Moreover, $B_{1}=B_{2}=0$. It means that both $q^{1}$ and $q^{2}$ are Ricci-flat. Then the solution is

$$
K=\left(\begin{array}{cc}
\frac{A}{n+2} z_{1}^{2}+\frac{E_{1}}{z_{1}^{n}} & \frac{A}{n+2} z_{1} z_{2}+\frac{F}{z_{1}^{n / 2} z_{2}^{n / 2}} \\
\frac{A}{n+2} z_{1} z_{2}+\frac{F}{z_{1}^{n / 2} z_{2}^{n / 2}} & \frac{A}{n+2} z_{2}^{2}+\frac{E_{2}}{z_{2}^{n}}
\end{array}\right) .
$$

However, we have not been able to decide the completeness of this metric.

Remark. We also worked on the constant scalar ansatz, then $A z_{i}+B_{i}$ is modified by adding a factor $\frac{G_{i}}{z_{i}^{n_{i}}}$. But such a system could have a solution only if $G_{i}=0$.

We have also obtained another Ricci-flat Kähler metric with $T^{N}$ symmetry without deciding the completeness. We still keep the geometrical assumptions which imply that the inner products $K_{i j}=g\left(X_{i}, X_{j}\right)$ only depend on the Hamiltonians $z_{i}$ and that

$$
h_{\mu \nu}=\left(C_{i} z_{i}+D\right) q_{\mu \nu}
$$

for constants $C_{i}, D$. Also,

$$
u=\log \operatorname{det} q_{\mu \nu}+n \log \left(C_{i} z_{i}+D\right)+\log \operatorname{det} K .
$$

If we furthermore assume that $K_{i j}$ is diagonal,

$$
K_{i j}=K I_{N}
$$

then (1.12) leads to the differential equations

$$
\frac{\partial}{\partial z_{k}}\left[\left(C_{i} z_{i}+D\right)^{n / N} K\right]=\frac{1}{N}\left(A z_{k}+B_{k}\right)\left(C_{i} z_{i}+D\right)^{n / N} .
$$

This is exact if we assume the metric has vanishing scalar curvature, i.e. $A=0$, and set $C_{k}=1$ and $B_{k}=\lambda$, for all $k=1, \ldots, N$.

Then we may solve (4.6) to get

$$
K=\frac{\lambda}{n+N}\left(\sum z_{i}\right)+\frac{E}{\left(\sum z_{i}\right)^{n / N}},
$$

where we have $D=0$. Now, again assuming that the metric $q$ is the Fubini-Study metric with constant holomorphic sectional curvature 1 the equation corresponding to (2.5) gives $\lambda=\frac{n}{N}(n+1)$. Then $d \theta_{k}=$ curvature of $\mathcal{O}(-2)$. Thus, the Ricci-flat Kähler metric is given by

$$
g=\left(\sum_{i=1}^{N} z_{i}\right) q+K^{-1} \sum_{i=1}^{N} d z_{i}^{2}+K \sum_{i=1}^{N}\left(d t_{i}+\theta_{i}\right)^{2}
$$


with

$$
K=\frac{n+1}{N(n+N)} \sum_{i=1}^{N} z_{i}+\frac{E}{\left(\sum z_{i}\right)^{n / N}} .
$$

For $N=1$, this is the Calabi metric.

One may also allow $I_{N}$ to be replaced by a positive definite symmetric matrix with constant coefficients. Then one obtains a family of Ricci-flat Kähler metrics.

Acknowledgements. We would like to thank C. LeBrun, K. P. Tod and the referee for valuable suggestions. Henrik Pedersen is grateful for the hospitality of the Department of Mathematics, Rice University, where this work began. Y. Sun Poon would like to thank the Department of Mathematics, Odense University, for their incredible hospitality when this paper was finished.

\section{References}

1. Besse, A. L.: Einstein Manifolds, Ergeb. Math. Grenzgeb. Vol. 10, Berlin, Heidelberg, New York: Springer 1987

2. Calabi, E.: The Space of Kähler metrics. Proc. Internat. Congress. Math. Amsterdam II 206-207 (1954)

3. Calabi, E.: Métriques Kähleriennes et Fibrés Holomorphes. Ann. Sci. Ec. Norm. Sup. $4^{\mathrm{eme}}$ série, 12, 269-294 (1979)

4. Calabi, E.: Extremal Kähler metrics, in seminar on differential geometry. Ann. Math. Stud. 102, 250-290 (1982), Princeton, NJ: Princeton Univ. Press

5. Futaki, A.: Kähler-Einstein Metrics and Integral Invariants. Lecture Notes in Math., Vol. 1314, Berlin, Heidelberg, New York: Springer 1980

6. LeBrun, C. R.: Counter-examples to the generalized positive action conjecture. Commun. Math. Phys. 118 591-596 (1988)

7. LeBrun, C. R.: Explicit Self-Dual Metrics on $\mathrm{CP}_{2} \# \cdots \# \mathrm{CP}_{2}$, Preprint, Princeton (1989)

8. LeBrun, C. R.: Anti-Self-Dual Hermitian Metrics on Blown-Up Hopf Surfaces. Preprint, Princeton (1989)

9. LeBrun, C. R.: Scalar-flat Kähler metrics on blown-up ruled surfaces. Preprint, Princeton (1989)

10. Pedersen, H.: Einstein metrics, spinning top motions and monopoles. Math. Annal. 274, 35-59 (1986)

11. Pedersen, N., Nielsen, B.: On some Euclidean Einstein Metrics. Lett. Math. Phys. 12, 277-282 (1986)

Communicated by S.-T. Yau 